\title{
Surgeon specialty and patient outcomes in carotid endarterectomy
}

\author{
Andreea Seicean, MD, PhD, MPH, ${ }^{1}$ Prateek Kumar, BA, ${ }^{2}$ Sinziana Seicean, MD, PhD, MPH, ${ }^{3}$ Duncan \\ Neuhauser, PhD, ${ }^{4}$ and Robert J. Weil, MD ${ }^{5}$
}

Departments of ${ }^{1}$ Psychiatry and ${ }^{2}$ Neurosurgery, University of Illinois at Chicago, Illinois; Departments of ${ }^{3}$ nternal Medicine and ${ }^{4}$ Epidemiology and Biostatistics, Case Western Reserve University, Cleveland, Ohio; and 5 National Clinical Enterprise, Catholic Health Initiatives, Englewood, Colorado

OBJECTIVE The goal of this study was to compare outcomes of carotid endarterectomy performed by neurological, general, and vascular surgeons.

METHODS The authors identified 80,475 patients who underwent carotid endarterectomy between 2006 and 2015 in the National Surgical Quality Improvement Program, a prospectively collected, national clinical database with established reproducibility and validity. Nine hundred forty-three patients were operated on by a neurosurgeon; 75,649 by a vascular surgeon; and 3734 by a general surgeon. Preoperative and intraoperative characteristics and 30 -day outcomes were stratified by the surgeon's primary specialty. Using propensity scores, comprising pre- and intraoperative characteristics as well as procedure and diagnostic codes, the authors matched 203 neurosurgery (NS) patients to 203 vascular surgery (VS) patients and 203 NS patients to 203 general surgery (GS) patients. No pre- or intraoperative factors were significantly different between specialties in the matched sample. Regular logistic regression and conditional logistic regression were used to predict postoperative complications in the full sample and in the matched sample.

RESULTS In the complete population sample, NS patients, when compared to patients of general and vascular surgeons, were less likely to be admitted from home and more likely to have carotid artery occlusion or stenosis with cerebral infarction, to be a current smoker, to have had recent chemo- or radiotherapy, to have surgery under general anesthesia, to undergo multiple procedures, and to have longer surgery times. In unadjusted analyses, NS patients were more likely to experience major complications (NS vs VS: odds ratio 1.3, 95\% Cl 1.1-1.6; NS vs GS: odds ratio $1.3,95 \%$ Cl 1.0-1.7); minor complications (NS vs VS: odds ratio 2.9, 95\% Cl 2.0-4.1; NS vs GS: odds ratio 2.7, 95\% Cl 1.7-4.2); intra- or postoperative transfusions (NS vs VS: odds ratio 1.6, 95\% Cl 1.4-1.9; NS vs GS: odds ratio 1.9, 95\% Cl 1.62.3); prolonged hospitalization (NS vs VS: odds ratio 3.0, $95 \% \mathrm{Cl} 2.6-3.5$; NS vs GS: odds ratio $2.6,95 \% \mathrm{Cl} 2.2-3.0$ ); and discharge to skilled care facilities (NS vs VS: odds ratio $2.8,95 \% \mathrm{Cl} 2.3-3.4$; NS vs GS: odds ratio $3.1,95 \% \mathrm{Cl}$ 2.4-4.1). In adjusted, propensity-matched analyses, however, patients' outcome with carotid endarterectomy performed by NS was comparable with those completed by GS and VS.

CONCLUSIONS Patients who undergo carotid endarterectomy performed by a neurosurgeon tend to have a greater preoperative disease burden than do those treated by a general or vascular surgeon, which contributes significantly to more morbid postoperative courses. In patients matched carefully on the basis of health status at the time of surgery and intraoperative variables that affect results, patients' outcomes after carotid endarterectomy do not appear to depend on the attending surgeon's primary specialty.

https://thejns.org/doi/abs/10.3171/2018.2.JNS173014

KEYWORDS comparative effectiveness; vascular surgery; general surgery; outcomes; neurosurgery; health services research; vascular disorders

ABBREVIATIONS BMI = body mass index; $\mathrm{CPR}=$ cardiopulmonary resuscitation; $\mathrm{CPT}=$ Current Procedural Terminology; $\mathrm{CVA}=$ cerebrovascular accident; $\mathrm{DVT}=$ deep venous thrombosis; GS = general surgery; ICD-9 = International Classification of Diseases, Ninth Revision; INR = international normalized ratio; LOS = length of hospital stay; MI = myocardial infarction; NS = neurosurgery; NSQIP = National Surgical Quality Improvement Program; OR = operating room; TIA = transient ischemic attack; VS = vascular surgery.

SUBMITTED November 29, 2017. ACCEPTED February 22, 2018.

INCLUDE WHEN CITING Published online August 10, 2018; DOI: 10.3171/2018.2.JNS173014. 
$\mathrm{N}$ EUROLOGICAL, vascular, and general surgeons perform carotid endarterectomy. There are differences in training between the 3 specialties in the amount of exposure and scope of practice in general as well as with respect to carotid endarterectomy. Although several studies have looked at differences in outcomes between surgical specialty and carotid endarterectomy, each has suffered from significant limitations in design, which include small sample sizes, single-institution designs, and most importantly, failure to control for patient and intraoperative characteristics. ${ }^{1,12,18,19,22,30}$ The combination of these limitations and the studies' divergent findings have resulted in a murky picture. We used the National Surgical Quality Improvement Program (NSQIP) database, a prospectively collected, clinical database, with proven validity and reproducibility, ${ }^{19,21}$ to conduct a comparative effectiveness study to analyze 30-day outcomes and the incidence of perioperative complications after carotid endarterectomy performed by a neurosurgeon, vascular surgeon, and general surgeon. Our large sample size and meticulous methods for controlling patient and operative characteristics allow for a more complete understanding of outcomes.

\section{Methods \\ Data Source}

We used the American College of Surgeons' NSQIP database to identify patients who underwent carotid endarterectomy between 2006 and 2015. This database consists of prospectively collected, clinical data from 603 community and academic hospitals in the US. Data consist of 274 variables, including demographics, preoperative laborato- ry values, comorbidities, intraoperative characteristics, and postoperative morbidity and mortality at 30 days. Hospitals that participate in the NSQIP undergo annual audits of interrater reliability to ensure accurate data collection. The NSQIP is a high-quality, reliable database..$^{4,21,31,33}$

\section{Subjects and Surgical Specialty}

Each patient has one primary Current Procedural Terminology (CPT) code, and up to 10 secondary CPT codes. Patients were considered to have undergone carotid endarterectomy if they had a CPT code of 35301 (thromboendarterectomy procedures on arteries and veins) and an International Classification of Diseases, Ninth Revision (ICD-9) code of 433.10 (occlusion and stenosis of carotid artery without cerebral infarction) or 433.11 (occlusion and stenosis of carotid artery with cerebral infarction). We identified 80,475 patients who underwent carotid endarterectomy between 2006 and 2015 (Fig. 1). Exclusion criteria included patients with septic shock $(n=3)$, and preoperative transfusion $(n=146)$, features that dictate a distinct postoperative course. ${ }^{5}$ Our study sample consists of 80,326 patients who underwent carotid endarterectomy. We stratified patients using the primary surgical specialty of the attending surgeon, with $943(1.2 \%)$ patients operated on by a neurosurgeon, $75,649(94.2 \%)$ by a vascular surgeon, and $3734(4.6 \%)$ by a general surgeon.

\section{Covariate Analysis}

We analyzed all available pre- and intraoperative factors in NSQIP that could influence postoperative outcomes (Table 1). ${ }^{31}$ Patient age, body mass index (BMI), and surgi-

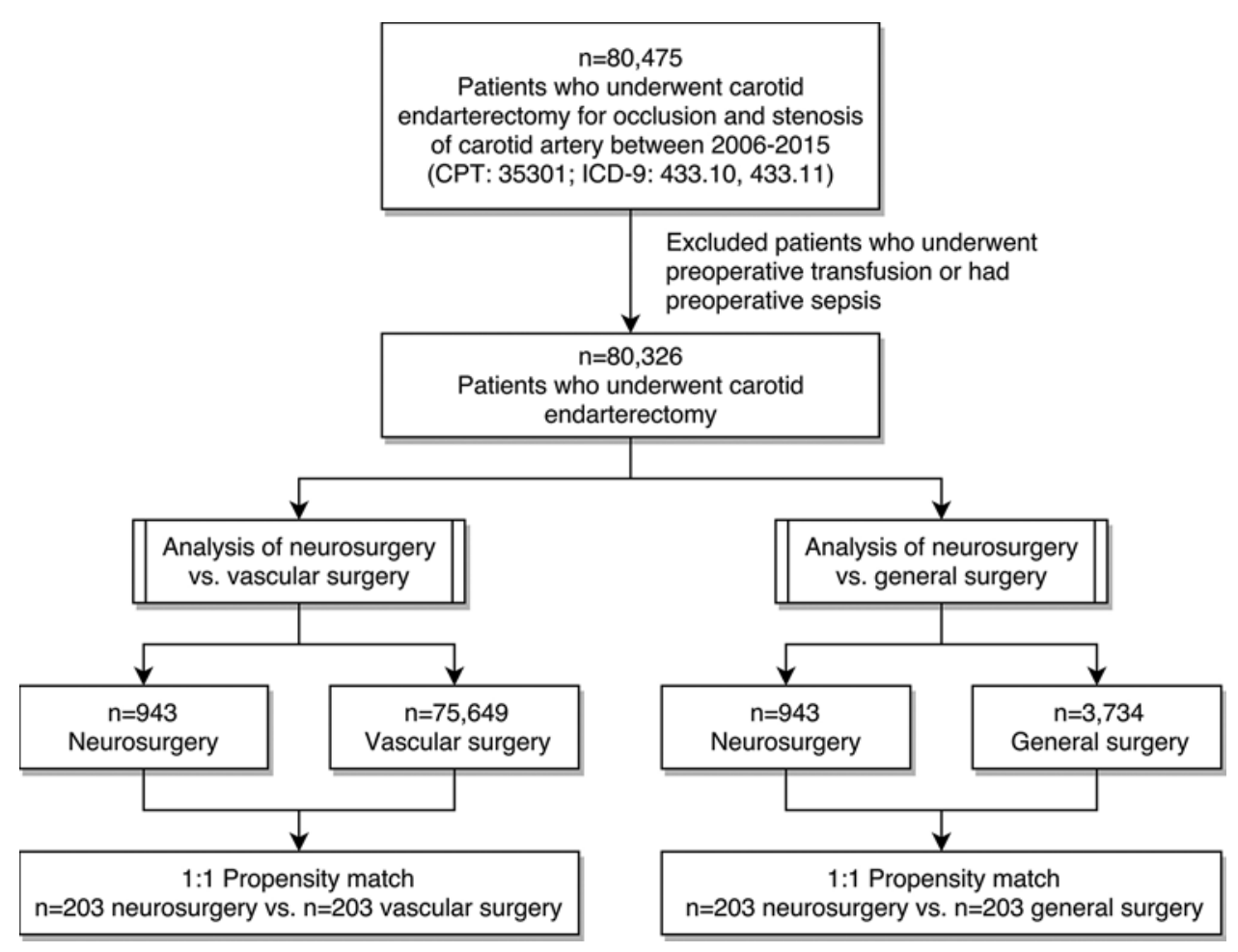

FIG. 1. Patient selection criteria: flow diagram demonstrating entry and exclusion criteria and breakdown of patients in the analyses. 
TABLE 1. Preoperative and intraoperative characteristics of patients who underwent carotid endarterectomy, according to surgical specialty $(\mathrm{N}=80,326)$

\begin{tabular}{|c|c|c|c|c|c|c|}
\hline Characteristic & NS $(n=943)$ & VS $(n=75,649)$ & ASD* & GS $(n=3734)$ & ASD† & ASD $\ddagger$ \\
\hline Age, yrs; mean \pm SD & $69 \pm 10$ & $71 \pm 9$ & 0.21 & $71 \pm 9$ & 0.24 & 0.03 \\
\hline Female & $34.0 \%$ & $40.2 \%$ & 0.13 & $41.7 \%$ & 0.14 & 0.03 \\
\hline Caucasian & $86.3 \%$ & $88.5 \%$ & 0.07 & $84.4 \%$ & 0.09 & 0.12 \\
\hline Admitted from home & $81.8 \%$ & $95.9 \%$ & 0.46 & $97.3 \%$ & 0.43 & 0.08 \\
\hline \multicolumn{7}{|l|}{ Smoking status } \\
\hline Current & $64.3 \%$ & $58.5 \%$ & \multirow[t]{3}{*}{0.30} & $60.7 \%$ & \multirow[t]{3}{*}{0.18} & \multirow[t]{3}{*}{0.08} \\
\hline Previous & $30.6 \%$ & $27.7 \%$ & & $28.2 \%$ & & \\
\hline Never & $5.1 \%$ & $13.8 \%$ & & $11.1 \%$ & & \\
\hline$>2$ alcoholic drinks per day & $3.5 \%$ & $4.2 \%$ & 0.04 & $3.9 \%$ & 0.02 & 0.02 \\
\hline Dependent functional status & $5.1 \%$ & $4.0 \%$ & 0.06 & $4.3 \%$ & 0.02 & 0.02 \\
\hline \multicolumn{7}{|l|}{ ASA classification } \\
\hline $1 \& 2$ & $10.4 \%$ & $7.3 \%$ & \multirow[t]{2}{*}{0.11} & $6.3 \%$ & \multirow[t]{2}{*}{0.18} & \multirow[t]{2}{*}{0.04} \\
\hline $3 \& 4$ & $89.6 \%$ & $92.7 \%$ & & $93.7 \%$ & & \\
\hline $\mathrm{BMI}, \mathrm{kg} / \mathrm{m}^{2} ;$ mean $\pm \mathrm{SD}$ & $29 \pm 6$ & $29 \pm 6$ & 0.05 & $29 \pm 6$ & 0.03 & 0.01 \\
\hline Hypertension requiring medication & $76.6 \%$ & $85.0 \%$ & 0.22 & $83.5 \%$ & 0.18 & 0.04 \\
\hline Diabetes mellitus & $26.6 \%$ & $29.3 \%$ & 0.06 & $30.3 \%$ & 0.07 & 0.02 \\
\hline Cerebrovascular comorbidities§ & $11.8 \%$ & $17.8 \%$ & 0.17 & $17.9 \%$ & 0.17 & $<0.01$ \\
\hline Cardiopulmonary comorbidities & $7.5 \%$ & $11.0 \%$ & 0.12 & $11.1 \%$ & 0.12 & $<0.01$ \\
\hline Renal comorbidities ${ }^{* *}$ & $28.2 \%$ & $37.8 \%$ & 0.21 & $37.8 \%$ & 0.20 & $<0.01$ \\
\hline Bleeding risk factors $† \dagger$ & $5.8 \%$ & $7.7 \%$ & 0.08 & $7.4 \%$ & 0.00 & 0.01 \\
\hline Chemo- \&/or radiotherapy & $2.8 \%$ & $0.2 \%$ & 0.49 & $0.1 \%$ & 0.62 & 0.04 \\
\hline Preop anemia & $30.0 \%$ & $32.8 \%$ & 0.07 & $34.2 \%$ & 0.11 & 0.07 \\
\hline Sepsis or SIRS & $2.0 \%$ & $0.7 \%$ & 0.12 & $0.5 \%$ & 0.18 & 0.02 \\
\hline Prior op w/in 30 days & $1.3 \%$ & $1.0 \%$ & 0.03 & $1.2 \%$ & 0.01 & 0.02 \\
\hline Abnormal WBC count & $12.6 \%$ & $10.8 \%$ & 0.06 & $10.7 \%$ & 0.00 & $<0.01$ \\
\hline 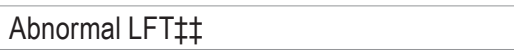 & $16.9 \%$ & $14.4 \%$ & 0.07 & $12.2 \%$ & 0.07 & 0.07 \\
\hline Abnormal sodium & $8.1 \%$ & $8.0 \%$ & 0.00 & $8.7 \%$ & 0.05 & 0.02 \\
\hline Length of op, min; mean \pm SD & $151 \pm 51$ & $115 \pm 47$ & 0.72 & $113 \pm 56$ & 0.70 & 0.04 \\
\hline General anesthesia & $95.7 \%$ & $86.3 \%$ & 0.33 & $86.4 \%$ & 0.33 & $<0.01$ \\
\hline Emergency & $3.5 \%$ & $1.7 \%$ & 0.11 & $1.5 \%$ & 0.13 & 0.02 \\
\hline Resident in OR & $80.4 \%$ & $57.9 \%$ & 0.50 & $30.7 \%$ & 1.16 & 0.57 \\
\hline Multiple CPT codes & $18.8 \%$ & $6.9 \%$ & 0.36 & $4.1 \%$ & 0.48 & 0.13 \\
\hline \multicolumn{7}{|l|}{ Diagnosis§§ } \\
\hline $\begin{array}{l}\text { Occlusion \& stenosis of carotid artery } \\
\text { w/o cerebral infarction (ICD-9 433.10) }\end{array}$ & $71.2 \%$ & $93.4 \%$ & \multirow[t]{2}{*}{0.61} & $94.3 \%$ & \multirow[t]{2}{*}{0.64} & \multirow[t]{2}{*}{0.04} \\
\hline $\begin{array}{l}\text { Occlusion \& stenosis of carotid artery w/ } \\
\text { cerebral infarction (ICD-9 433.11) }\end{array}$ & $29.8 \%$ & $6.6 \%$ & & $5.7 \%$ & & \\
\hline
\end{tabular}

ASA = American Society of Anesthesiologists; ASD = absolute standardized difference; LFT = liver function test; SIRS = systemic inflammatory response syndrome; $\mathrm{WBC}=$ white blood cell.

Absolute value of the standard difference $>0.2$ indicates covariate imbalance (boldface type).

* Neurosurgery compared to vascular surgery.

$\dagger$ Neurosurgery compared to general surgery.

$\ddagger$ General surgery compared to vascular surgery.

$\S$ History of TIA or CVA with or without residual neurological deficits.

Tा Diagnosis of pulmonary and/or cardiovascular disease, or dyspnea.

** Diagnosis of renal disease, abnormal blood urea nitrogen, or creatinine.

†† Diagnosis of bleeding diathesis, abnormal INR, or abnormal platelet count.

拉 Abnormal bilirubin, alkaline phosphatase, aspartate transaminase, or albumin.

$\S \S$ ICD-9 diagnostic codes. 
TABLE 2. Distribution of 30-day postoperative outcomes in carotid endarterectomy patients, according to surgical specialty $(\mathrm{N}=80,326)$

\begin{tabular}{|c|c|c|c|}
\hline Outcome & $\begin{array}{c}\text { NS } \\
(n=943)\end{array}$ & $\begin{array}{c}V S \\
(n=75,649)\end{array}$ & $\begin{array}{c}\text { GS } \\
(n=3734)\end{array}$ \\
\hline \multicolumn{4}{|l|}{ Total LOS, days } \\
\hline Mean \pm SD & $4 \pm 6$ & $3 \pm 5$ & $2 \pm 3$ \\
\hline Median & 2 & 1 & 1 \\
\hline Prolonged LOS (>2 days) & $66.9 \%$ & $40.2 \%$ & $43.9 \%$ \\
\hline Minor complications* & $3.5 \%$ & $1.3 \%$ & $1.3 \%$ \\
\hline Superficial site infection & $0.4 \%$ & $0.3 \%$ & $0.4 \%$ \\
\hline Urinary tract infection & $2.6 \%$ & $0.8 \%$ & $0.9 \%$ \\
\hline DVT or thrombophlebitis & $0.6 \%$ & $0.2 \%$ & $0.1 \%$ \\
\hline Major complications $\dagger$ & $9.0 \%$ & $7.0 \%$ & $7.0 \%$ \\
\hline $\begin{array}{l}\text { Deep incision surgical site } \\
\text { infection }\end{array}$ & $0.3 \%$ & $0.1 \%$ & $0.1 \%$ \\
\hline Pneumonia & $1.7 \%$ & $0.8 \%$ & $1.1 \%$ \\
\hline Unplanned intubation & $2.9 \%$ & $1.1 \%$ & $1.3 \%$ \\
\hline $\begin{array}{l}>48 \text { hrs of ventilator-assist- } \\
\text { ed respiration }\end{array}$ & $2.4 \%$ & $0.7 \%$ & $0.6 \%$ \\
\hline Pulmonary embolism & $0.4 \%$ & $0.8 \%$ & $0 \%$ \\
\hline Renal insufficiency or failure & $0.5 \%$ & $0.2 \%$ & $0.4 \%$ \\
\hline CVA w/ neurological deficit & $2.9 \%$ & $1.5 \%$ & $2.0 \%$ \\
\hline Coma of $>24 \mathrm{hrs}$ & $0.1 \%$ & $0 \%$ & $0.1 \%$ \\
\hline Peripheral nerve injury & $0 \%$ & $0.1 \%$ & $0.1 \%$ \\
\hline Cardiac arrest or Ml & $1.6 \%$ & $1.2 \%$ & $0.9 \%$ \\
\hline Sepsis or septic shock & $0.9 \%$ & $0.5 \%$ & $0.3 \%$ \\
\hline Any complications $\ddagger$ & $11.0 \%$ & $7.8 \%$ & $7.9 \%$ \\
\hline Intra- or postop transfusion & $18.0 \%$ & $11.9 \%$ & $10.4 \%$ \\
\hline Discharged w/ continued care§ & $14.7 \%$ & $5.8 \%$ & $5.2 \%$ \\
\hline Return to OR w/in 30 days & $3.8 \%$ & $3.7 \%$ & $3.3 \%$ \\
\hline Readmission w/in 30 days & $6.3 \%$ & $7.0 \%$ & $7.0 \%$ \\
\hline 30-day mortality & $0.9 \%$ & $0.7 \%$ & $0.9 \%$ \\
\hline
\end{tabular}

${ }^{*}$ Having any one or more of the following complications within 30 days postoperatively: superficial surgical site infection, urinary tract infection, DVT, or thrombophlebitis.

† Having any one or more of the following complications within 30 days postoperatively: deep incision surgical site infection, organ or space surgical site infection, wound disruption, pneumonia, unplanned intubation, pulmonary embolism, $>48$ hours of postoperative ventilator-assisted respiration, progressive renal insufficiency, acute renal failure, cardiovascular accident with neurological deficit, coma of $>24$ hours, cardiac arrest requiring CPR, MI, sepsis, septic shock, and/or 30-day return to the OR.

$\ddagger$ Having any of 1 or more minor or major complications.

$\S$ Being discharged to continued care facility, unless patient was originally admitted from such a facility.

cal duration were included as continuous variables. ${ }^{31} \mathrm{We}$ combined race categories into white versus all others. ${ }^{31}$ We dichotomized transfer status as admitted from home versus transferred from any facility. We dichotomized functional status as independent versus partially or totally dependent. ${ }^{31}$ We defined altered mental status as acute mental status changes and/or delirium at the time of surgery. We classified patients who had a history of transient ischemic attacks (TIAs) or cerebrovascular accident
(CVA) with or without residual neurological deficits as having cerebrovascular comorbidities. Patients were considered to have cardiopulmonary comorbidities if they had any of the following: ventilator-assisted respiration during the 48 hours prior to surgery; congestive heart failure that was diagnosed or was symptomatic within 30 days prior to surgery; self-reported angina in the month leading up to surgery; myocardial infarction (MI) within the 6 months prior to surgery; any history of percutaneous coronary intervention, prior cardiac surgery, angioplasty, or revascularization procedure for atherosclerotic peripheral vascular disease; or were experiencing resting pain or gangrene.

Preoperative hemostatic screening laboratory values for blood drawn within 90 days of surgery were recorded in the NSQIP, and were considered abnormal using commonly accepted guidelines. ${ }^{23}$ Renal comorbidities were defined as patients with renal disease or abnormal blood urea nitrogen or creatinine lab values. Cancer comorbidities were defined as patients with unintentional weight loss $>10 \%$ of body weight in the 6 months prior to surgery, disseminated cancer, or receiving chemotherapy or radiotherapy within 90 days of surgery. The NSQIP variable "bleeding disorders" captures self-reported history of abnormal bleeding, family history of bleeding disorders, vitamin $\mathrm{K}$ deficiency, and a comprehensive list of medications that pose a risk for bleeding abnormalities disorder. Patients with abnormal platelet count, abnormal preoperative international normalized ratio (INR), or bleeding disorders were considered to have bleeding risk factors. Anemia was defined as hematocrit $<36 \%$ and $<41 \%$ in women and men, respectively. Abnormal bilirubin, alkaline phosphatase, aspartate transaminase, or albumin was used to create the variable abnormal liver function tests. Data on some comorbidities were only available for the years 2006-2013. We used the presence of resident physicians during the operation as a surrogate marker for academic institutions. "Multiple CPT codes" captures patients who underwent more than one procedure and hence had more than one CPT code. Covariate methodology has been previously described elsewhere by the authors. ${ }^{31}$

\section{Outcomes of Interest}

Outcomes of interest (Table 2) were as follows: 1) length of hospital stay (LOS), as a continuous variable; 2) prolonged LOS, which we chose to define as postoperative hospitalization longer than the third quartile of the study population, which here was $>2$ days; 3 ) minor postoperative complications defined as one or more of the following: superficial surgical site infection, urinary tract infection, deep venous thrombosis (DVT), or thrombophlebitis; 4) major postoperative complications defined as one or more of the following: deep incision surgical site infection, organ or space surgical site infection, or wound disruption; pneumonia, unplanned intubation, pulmonary embolism, or $>48$-hour postoperative ventilator-assisted respiration; progressive renal insufficiency or acute renal failure; cardiovascular accident with neurological deficit, coma of $>24$ hours, peripheral nerve injury, cardiac arrest requiring cardiopulmonary resuscitation (CPR), MI, graft, prosthesis or flap failure; sepsis or septic shock; and/or 30day return to the operating room (OR); 5) any postopera- 
TABLE 3. Association between surgical specialty and perioperative complications in patients undergoing carotid endarterectomy

\begin{tabular}{|c|c|c|c|c|c|c|}
\hline \multirow[b]{3}{*}{ Outcome* } & \multicolumn{6}{|c|}{ Odds Ratio $(95 \% \mathrm{Cl})$} \\
\hline & \multicolumn{2}{|c|}{ NS vs VS } & \multicolumn{2}{|c|}{ NS vs GS } & \multicolumn{2}{|c|}{ GS vs VS } \\
\hline & General† & Matched $\ddagger$ & General† & Matchedf & General† & Matched $\neq$ \\
\hline Prolonged LOS (>2 days) & $3.0(2.6-3.5)$ & $1.7(1.1-2.5)$ & $2.6(2.2-3.0)$ & $1.1(0.7-1.6)$ & $1.2(1.1-1.2)$ & $1.5(1.3-1.7)$ \\
\hline Minor complications & $2.9(2.0-4.1)$ & $2.0(0.6-6.6)$ & $2.7(1.7-4.2)$ & $3.5(0.7-16.8)$ & $1.0(0.8-1.4)$ & $1.0(0.6-1.7)$ \\
\hline Major complications & $1.3(1.1-1.6)$ & $0.5(0.2-1.1)$ & $1.3(1.0-1.7)$ & $0.9(0.4-2.0)$ & $1.0(0.9-1.1)$ & $1.1(0.8-1.3)$ \\
\hline Any complications & $1.5(1.2-1.8)$ & $0.8(0.4-1.6)$ & $1.5(1.1-1.8)$ & $1.2(0.6-2.5)$ & $1.0(0.9-1.1)$ & $1.1(0.9-1.3)$ \\
\hline Intra- or postop transfusion & $1.6(1.4-1.9)$ & $0.8(0.2-3.4)$ & $1.9(1.6-2.3)$ & $0.4(0.1-1.4)$ & $0.9(0.8-1.0)$ & $1.3(0.8-2.1)$ \\
\hline Discharged w/ continued care & $2.8(2.3-3.4)$ & NA & $3.1(2.4-4.1)$ & NA & $0.9(0.7-1.1)$ & $3.0(0.3-28.8)$ \\
\hline Return to OR w/in 30 days & $1.0(0.7-1.5)$ & $0.2(0.1-0.7)$ & $1.2(0.8-1.7)$ & $0.4(0.1-1.4)$ & $0.9(0.7-1.1)$ & $1.0(0.7-1.4)$ \\
\hline Readmission w/in 30 days & $0.9(0.7-1.2)$ & $1.0(0.1-16.0)$ & $0.9(0.6-1.2)$ & NA & $1.0(0.8-1.2)$ & $1.4(0.4-4.4)$ \\
\hline 30-day mortality & $1.3(0.6-2.6)$ & NA & $1.0(0.5-2.2)$ & $0.8(0.2-3.4)$ & $1.3(0.9-1.9)$ & $1.2(0.6-2.5)$ \\
\hline
\end{tabular}

$\mathrm{NA}=$ not available because logistic model may be inaccurate due to low frequency of this outcome in the matched sample.

Boldface type indicates statistically significant odds ratios.

* See Table 2 for description of outcomes.

† Logistic regression.

$\ddagger$ Conditional logistic regression, which takes into account the matched nature of the analysis.

tive complication, defined as having one or more minor or major complication or an unplanned return to the OR; 6) discharged with continued care, defined as discharged to home with continued care or to a skilled or unskilled care facility but who had not been admitted from such a facility; 7) unplanned return to the OR within 30 days of surgery; 8) 30-day readmission, defined as readmission to hospital within 30 days of discharge; and 9) 30-day mortality, defined as death within 30 days after index surgery regardless of hospitalization status.

\section{Statistical Analyses}

We used standardized differences to compare preand intraoperative factors according to surgeon specialty (Table 1). This is the ideal tool to assess intragroup differences in covariate balance because standardized differences are not affected by sample size, unlike significance tests such as Pearson's chi-square or Fisher's test, which generate $\mathrm{p}$ values. ${ }^{6}$ This is important in matched analyses, where the invariably smaller sample size of the matched cohort may result in statistically insignificant $p$ values that are falsely interpreted as improved covariate balance. ${ }^{31} \mathrm{An}$ absolute standardized difference of $>0.20$ was considered statistically significant. ${ }^{7}$ For example, we found that age, location admitted from, smoking status, cardiopulmonary comorbidities, renal comorbidities, chemo- or radiotherapy, length of surgery, presence of a resident in the OR, multiple CPT codes, and diagnosis were significantly different between neurosurgery (NS) and vascular surgery (VS) patients.

Given the nonrandomized design of this study, our best option to control for imbalance with regard to these covariates was to generate a propensity score that included all pre- and intraoperative characteristics that were significantly different between NS and VS patients, diagnostic code, and procedure performed. ${ }^{6,27}$ We used 1:1 greedy matching analyses ${ }^{8,26,27}$ to match NS patients with VS patients according to their respective propensity score. In greedy matching, an NS patient is selected at random and matched to a VS patient whose propensity score is closest to that of the NS patient. ${ }^{26}$ This process was used until all NS patients were matched to VS patients, and then was repeated with the other specialty comparisons. We successfully matched 203 NS patients to 203 VS patients to create our matched cohort. To ensure that covariate balance was achieved with propensity score matching, we compared baseline characteristics of patients in the matched cohort by using standardized difference. We no longer found any covariate imbalance. This same process was repeated to successfully match 203 NS patients to 203 general surgery (GS) patients, with no covariate imbalance following the match.

We used logistic regression analysis to assess whether surgical specialty was independently associated with adverse outcomes in the unmatched cohort (Table 3). We used conditional logistic regression analysis to model the relationship between surgical subspecialty and adverse outcomes in the matched cohort. ${ }^{6,11}$ SAS software (version 9.4, SAS Institute) was used. This study was approved by the University of Illinois at Chicago Institutional Review Board.

\section{Results}

Preoperative and intraoperative characteristics are listed in Table 1 according to surgical specialty. A number of pre- and intraoperative factors were significantly different between surgical subspecialties. Compared to both VS and GS patients, NS patients were significantly more likely to have occlusion and stenosis of the carotid artery with (as opposed to without) cerebral infarction (NS $29.8 \%$, VS $6.6 \%$, GS $5.7 \%$ ). They were also more likely to have carotid endarterectomy performed under general anesthesia, to undergo multiple procedures, and to have longer surgery times. NS patients were less likely to be admitted from home and more likely to be current smok- 
ers and to have had recent chemo- or radiotherapy. On the other hand, VS and GS patients more often had renal comorbidities and hypertension requiring medication(s). VS and GS patients were slightly older than NS patients (mean age 71 vs 69 years).

Outcome frequency was compared between NS, VS, and GS patients (Table 2). The mean length of hospitalization in NS patients was 4 days, versus 3 days in VS and 2 days in GS patients. Prolonged hospitalization, intra- or postoperative transfusions, complications, and need for continued care after discharge were more prevalent in NS patients. However, the rates of 30-day readmission, 30-day return to the OR, and 30-day mortality were similar or lower in NS patients than in VS and GS patients.

We matched 203 NS to 203 VS patients by using propensity scores (Table 4). There is no covariate imbalance with regard to pre- and intraoperative factors, preoperative diagnoses, or operative procedures between surgical specialties in the matched sample. Table 5 shows the frequencies of outcomes in the matched sample.

Using logistic regression in the general, unmatched cohort of 80,326 patients (Table 3), we found that compared to VS and GS, the NS cohort had slightly increased odds for major complications and intra- or postoperative transfusions, and nearly 3 times the odds for minor complications, prolonged LOS, and discharge with continued care. We used conditional logistic regression to assess the relationship between surgical specialty and each outcome of interest in the matched cohort. Once matched for pre- and intraoperative characteristics, there was no difference in outcomes between NS, VS, and GS patients.

Sensitivity analyses were done separating patients who presented with preoperative TIA or CVA. This did not change our results (data not shown).

\section{Discussion}

The NSQIP database is a large, clinical, prospectively collected database, with data collected from more than 600 hospitals from around the US to conduct a comparative effectiveness study of patients undergoing carotid endarterectomy. In our analysis of more than 80,000 patients, NS patients were more likely than VS and GS patients to undergo endarterectomy for occlusion and stenosis of the carotid artery with cerebral infarction, to have surgery with general anesthesia, to undergo multiple procedures, and to have longer surgery times. In the full population, NS patients had increased odds of complications, transfusions, prolonged LOS, and discharge with continued care. In the propensity-matched sample, which controlled for baseline and intraoperative patient factors, no differences in outcomes were found.

\section{In the Context of the Literature}

Rates of postoperative outcomes found in this study were similar to 30-day outcomes of large prospective clinical trials., ${ }^{9,1425,28}$ Other studies have compared outcomes of carotid endarterectomy between various specialties including NS, VS, GS, and cardiothoracic surgery, but they have reported contrasting results. Several have suggested that patients treated with VS have lower rates of adverse events compared with other specialties, ${ }^{1,12,17,18,29,32}$ and that patients who undergo NS have increased durations of operations and hospital stays. ${ }^{22,24,29}$ Others claim that although morbidity rates differ, mortality rates do not; ${ }^{-1,12,18,22}$ still others claim no differences in morbidity and mortality altogether. ${ }^{10,16,20}$ These studies have been limited by small sample sizes and single-institution designs, but most importantly, they have not controlled for patient characteristics, surgical procedures, and intraoperative characteristics. No study has performed matched analyses that allow for direct comparison of NS, GS, and VS patients with equivalent diagnoses, equivalent procedures, and analogous baseline and intraoperative characteristics.

Two prior studies used the NSQIP database: Enomoto et al. studied 34,493 operations performed by vascular and general surgeons, and Lieber et al. studied 42,369 operations performed by neurosurgeons, vascular surgeons, general surgeons, and cardiothoracic surgeons. ${ }^{12,22}$ Enomoto et al. did conduct propensity score-matched analyses and concluded that after controlling for patient and surgical characteristics, there was no difference in LOS or mortality rates between VS and GS, but that GS had lower risks of MI and VS had lower risks of stroke and infection. ${ }^{12}$ Unlike Enomoto et al., Lieber et al. included NS patients in their analysis and found that whereas mortality rates were not significantly different between specialties, NS patients had significantly greater rates of venous thromboembolism and postoperative requirement for ventilator-assisted respiration $>48$ hours..$^{12,22}$ Our study, by ensuring covariate balance and controlling for the increased preoperative morbidity of NS patients, found that these differencesand, in fact, all differences-in outcomes lost significance after the matching process. With the largest sample size by far and with the most controlled data analyses, our study allows for a more accurate understanding of outcomes after carotid endarterectomy.

Various studies are in disagreement and show controversy as to the effect that surgeon and hospital volume could have on carotid endarterectomy outcomes. , $29,30,34^{2}$ Also, there is disagreement about methodological approaches to assess the relationship between volume and outcomes..$^{15}$ There are no data on hospital and surgeon volume available in the NSQIP. Several studies suggest that volume is a stronger predictor of patient outcomes than surgical specialty.1,10,13,29 The NSQIP, however, does not contain any institutional identifiers, including data to show which patients underwent operation at the same institution or by the same surgeon. We used resident presence during surgery as a surrogate for academic institutions.

\section{Clinical Implications}

In the current analysis of more than 80,000 patients in whom carotid endarterectomy was performed by neurosurgeons, vascular surgeons, and general surgeons, we found significant differences in outcomes, with neurosurgeons having slightly increased odds for major complications and intra- or postoperative transfusions, and nearly 3 times the odds for minor complications, prolonged LOS, and discharge with continued care. However, NS patients tend to have a greater disease burden at the time of surgery, including occlusion and stenosis of the carotid artery 
TABLE 4. Baseline characteristics of patients who underwent carotid endarterectomy in the matched sample, according to surgical specialty

\begin{tabular}{|c|c|c|c|c|c|c|c|c|c|}
\hline Characteristic & $N S(n=203)$ & VS $(n=203)$ & $\mathrm{ASD}^{*}$ & NS $(n=203)$ & $G S(n=203)$ & ASD† & VS $(n=1978)$ & GS $(n=1978)$ & ASD \\
\hline Age, yrs; mean \pm SD & $70 \pm 11$ & $71 \pm 9$ & 0.11 & $70 \pm 11$ & $70 \pm 10$ & 0.05 & $71 \pm 9$ & $71 \pm 9$ & 0.01 \\
\hline Female & $32.5 \%$ & $36.5 \%$ & 0.08 & $32.5 \%$ & $36.9 \%$ & 0.09 & $40.1 \%$ & $42.7 \%$ & 0.05 \\
\hline Caucasian & $85.6 \%$ & $91.4 \%$ & 0.18 & $85.6 \%$ & $82.3 \%$ & 0.09 & $88.6 \%$ & $82.5 \%$ & 0.17 \\
\hline Admitted from home & $91.1 \%$ & $91.1 \%$ & 0 & $91.1 \%$ & $92.6 \%$ & 0.05 & $96.5 \%$ & $97.2 \%$ & 0.04 \\
\hline \multicolumn{10}{|l|}{ Smoking status } \\
\hline Current & $19.7 \%$ & $19.7 \%$ & \multirow[t]{3}{*}{0.01} & $19.7 \%$ & $19.7 \%$ & \multirow[t]{3}{*}{0.04} & $22.2 \%$ & $19.2 \%$ & \multirow[t]{3}{*}{0.08} \\
\hline Previous & $27.1 \%$ & $26.6 \%$ & & $27.1 \%$ & $25.6 \%$ & & $27.4 \%$ & $28.1 \%$ & \\
\hline Never & $53.2 \%$ & $53.7 \%$ & & $53.2 \%$ & $54.7 \%$ & & $50.4 \%$ & $52.8 \%$ & \\
\hline$>2$ alcoholic drinks per day & $3.9 \%$ & $4.9 \%$ & 0.05 & $3.9 \%$ & $5.4 \%$ & 0.07 & $4.6 \%$ & $4.0 \%$ & 0.03 \\
\hline Dependent functional status & $7.4 \%$ & $6.9 \%$ & 0.02 & $7.4 \%$ & $5.9 \%$ & 0.06 & $5.0 \%$ & $5.2 \%$ & 0.01 \\
\hline \multicolumn{10}{|l|}{ ASA classification } \\
\hline $1 \& 2$ & $13.9 \%$ & $8.9 \%$ & \multirow[t]{2}{*}{0.16} & $13.9 \%$ & $11.3 \%$ & \multirow[t]{2}{*}{0.08} & $8.4 \%$ & $7.7 \%$ & \multirow[t]{2}{*}{0.02} \\
\hline $3 \& 4$ & $86.1 \%$ & $91.1 \%$ & & $86.1 \%$ & $88.7 \%$ & & $91.7 \%$ & $92.3 \%$ & \\
\hline $\mathrm{BMI}, \mathrm{kg} / \mathrm{m}^{2} ;$ mean $\pm \mathrm{SD}$ & $28 \pm 5$ & $29 \pm 6$ & 0.02 & $28 \pm 5$ & $28 \pm 6$ & 0.06 & $29 \pm 6$ & $29 \pm 6$ & 0.00 \\
\hline Hypertension requiring medication & $78.8 \%$ & $81.8 \%$ & 0.07 & $78.8 \%$ & $79.3 \%$ & 0.01 & $86.5 \%$ & $84.5 \%$ & 0.06 \\
\hline Diabetes mellitus & $23.2 \%$ & $22.2 \%$ & 0.02 & $23.2 \%$ & $22.2 \%$ & 0.02 & $28.0 \%$ & $28.8 \%$ & 0.02 \\
\hline Cerebrovascular comorbidities§ & $42.9 \%$ & $39.4 \%$ & 0.07 & $42.9 \%$ & $34.0 \%$ & 0.18 & $35.2 \%$ & $32.3 \%$ & 0.06 \\
\hline 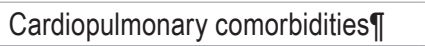 & $5.9 \%$ & $8.9 \%$ & 0.11 & $5.9 \%$ & $7.9 \%$ & 0.08 & $11.6 \%$ & $10.4 \%$ & 0.04 \\
\hline Renal comorbidities** & $19.2 \%$ & $19.2 \%$ & 0 & $19.2 \%$ & $20.2 \%$ & 0.02 & $36.5 \%$ & $39.4 \%$ & 0.06 \\
\hline Bleeding risk factors $† \dagger$ & $5.5 \%$ & $6.5 \%$ & 0.04 & $5.5 \%$ & $6.9 \%$ & 0.06 & $7.8 \%$ & $7.6 \%$ & 0.00 \\
\hline Chemo- \&/or radiotherapy & $0.5 \%$ & $0.5 \%$ & 0.07 & $0.5 \%$ & $0.5 \%$ & 0.18 & $0.3 \%$ & $0.2 \%$ & 0.16 \\
\hline Preop anemia & $26.1 \%$ & $26.6 \%$ & 0.01 & $26.1 \%$ & $32.5 \%$ & 0.18 & $34.1 \%$ & $36.0 \%$ & 0.05 \\
\hline Sepsis or SIRS & $2.5 \%$ & $0.5 \%$ & 0.16 & $2.5 \%$ & $0.5 \%$ & 0.16 & $0.8 \%$ & $0.2 \%$ & 0.08 \\
\hline Prior op w/in 30 days & $1.5 \%$ & $4.2 \%$ & 0.16 & $1.5 \%$ & $1.1 \%$ & 0.04 & $0.9 \%$ & $1.2 \%$ & 0.03 \\
\hline Abnormal WBC count & $7.2 \%$ & $13.2 \%$ & 0.20 & $7.2 \%$ & $11.7 \%$ & 0.15 & $11.1 \%$ & $10.3 \%$ & 0.02 \\
\hline Abnormal LFTł‡ & $13.9 \%$ & $13.4 \%$ & 0.02 & $13.9 \%$ & $14.1 \%$ & 0.00 & $14.3 \%$ & $11.2 \%$ & 0.10 \\
\hline Abnormal sodium & $5.0 \%$ & $9.2 \%$ & 0.16 & $5.0 \%$ & $7.0 \%$ & 0.08 & $8.3 \%$ & $8.1 \%$ & 0.01 \\
\hline Length of op, min; mean \pm SD & $150 \pm 47$ & $118 \pm 41$ & 0.70 & $150 \pm 47$ & $141 \pm 77$ & 0.14 & $108 \pm 44$ & $118 \pm 63$ & 0.19 \\
\hline General anesthesia & $84.2 \%$ & $84.7 \%$ & 0.01 & $84.2 \%$ & $86.2 \%$ & 0.05 & $84.6 \%$ & $86.5 \%$ & 0.05 \\
\hline Intra- or postop transfusion & $1.5 \%$ & $2.0 \%$ & 0.04 & $1.5 \%$ & $4.0 \%$ & 0.15 & $1.6 \%$ & $2.1 \%$ & 0.04 \\
\hline Emergency & $5.9 \%$ & $3.9 \%$ & 0.09 & $5.9 \%$ & $3.0 \%$ & 0.14 & $1.2 \%$ & $1.4 \%$ & 0.01 \\
\hline Resident in OR & $80.3 \%$ & $80.3 \%$ & 0 & $80.3 \%$ & $79.3 \%$ & 0.14 & $30.7 \%$ & $30.7 \%$ & 0 \\
\hline Multiple CPT codes & $8.4 \%$ & $7.9 \%$ & 0.02 & $8.4 \%$ & $7.4 \%$ & 0.04 & $7.0 \%$ & $4.1 \%$ & 0.13 \\
\hline \multicolumn{10}{|l|}{ Diagnosis§§ } \\
\hline $\begin{array}{l}\text { Occlusion \& stenosis of carotid } \\
\text { artery w/o cerebral infarction }\end{array}$ & $79.8 \%$ & $79.8 \%$ & \multirow[t]{2}{*}{0} & $79.8 \%$ & $81.3 \%$ & \multirow[t]{2}{*}{0.04} & $95.3 \%$ & $95.5 \%$ & \multirow[t]{2}{*}{0.01} \\
\hline $\begin{array}{l}\text { Occlusion \& stenosis of carotid } \\
\text { artery w/ cerebral infarction }\end{array}$ & $20.2 \%$ & $20.2 \%$ & & $20.2 \%$ & $18.7 \%$ & & $4.7 \%$ & $4.5 \%$ & \\
\hline
\end{tabular}

Absolute value of the standard difference $>0.2$ indicates covariate imbalance (boldface type).

* Neurosurgery compared to vascular surgery.

$†$ Neurosurgery compared to general surgery.

$\ddagger$ Vascular surgery compared to general surgery.

$\S$ History of TIAs or CVA with or without residual neurological deficits.

Tा Diagnosis of pulmonary and/or cardiovascular disease, or dyspnea.

** Diagnosis of renal disease, abnormal blood urea nitrogen, or creatinine.

†† Diagnosis of bleeding diathesis, abnormal INR, or abnormal platelet count.

$\ddagger \ddagger$ Abnormal bilirubin, alkaline phosphatase, aspartate transaminase, or albumin.

$\S \S I C D-9$ diagnostic codes. 
TABLE 5. Distribution of 30-day postoperative outcomes in the matched sample of carotid endarterectomy patients, according to surgical specialty

\begin{tabular}{|c|c|c|c|c|c|c|}
\hline Outcome & NS ( $n=203)$ & VS $(n=203)$ & $N S(n=203)$ & GS $(n=203)$ & VS $(n=1978)$ & GS $(n=1978)$ \\
\hline \multicolumn{7}{|l|}{ Total LOS, days } \\
\hline Mean \pm SD & $4 \pm 5$ & $3 \pm 4$ & $4 \pm 5$ & $3 \pm 5$ & $3 \pm 12$ & $3 \pm 4$ \\
\hline Median & 2 & 1 & 2 & 2 & 1 & 1 \\
\hline Prolonged LOS (>2 days) & $54.7 \%$ & $42.9 \%$ & $54.7 \%$ & $53.2 \%$ & $38.8 \%$ & $48.6 \%$ \\
\hline Minor complications ${ }^{*}$ & $2.0 \%$ & $3.9 \%$ & $3.9 \%$ & $1.5 \%$ & $1.3 \%$ & $1.3 \%$ \\
\hline Major complications $†$ & $6.9 \%$ & $11.3 \%$ & $6.9 \%$ & $7.4 \%$ & $7.7 \%$ & $8.1 \%$ \\
\hline Any complications $\ddagger$ & $9.9 \%$ & $11.8 \%$ & $9.9 \%$ & $8.4 \%$ & $8.3 \%$ & $8.9 \%$ \\
\hline Intra- or postop transfusion & $1.5 \%$ & $1.9 \%$ & $1.5 \%$ & $3.9 \%$ & $1.6 \%$ & $2.1 \%$ \\
\hline Return to the OR w/in 30 days & $1.5 \%$ & $6.9 \%$ & $1.5 \%$ & $3.9 \%$ & $4.2 \%$ & $4.3 \%$ \\
\hline Discharged w/ continued care§ & $10.3 \%$ & $11.1 \%$ & $10.3 \%$ & $9.5 \%$ & $6.4 \%$ & $4.8 \%$ \\
\hline Readmission w/in 30 days & $3.4 \%$ & $7.7 \%$ & $2.6 \%$ & $3.4 \%$ & $5.3 \%$ & $6.8 \%$ \\
\hline 30-day mortality & $1.5 \%$ & 0 & $1.5 \%$ & $2.0 \%$ & $0.7 \%$ & $0.9 \%$ \\
\hline
\end{tabular}

* Having any one or more of the following complications within 30 days postoperatively: superficial surgical site infection, urinary tract infection, DVT, or thrombophlebitis. $\dagger$ Having any one or more of the following complications within 30 days postoperatively: deep incision surgical site infection, organ or space surgical site infection, wound disruption, pneumonia, unplanned intubation, pulmonary embolism, $>48$ hours of postoperative ventilator-assisted respiration, progressive renal insufficiency, acute renal failure, cardiovascular accident with neurological deficit, coma of $>24$ hours, cardiac arrest requiring CPR, MI, sepsis, septic shock, and/or 30-day return to the OR.

‡ Having any 1 or more minor or major complications.

$\S$ Being discharged to continued care facility, unless patient was originally admitted from such a facility.

with cerebral infarction, having to undergo multiple procedures, and having longer surgery times compared with GS or VS patients, and this contributed significantly to the more morbid postoperative courses.

In equally healthy patients, matched carefully on the basis of significant preoperative characteristics and morbidities, the outcome of carotid endarterectomy does not appear to depend on surgical specialty. Once the patients were accurately paired based on preoperative characteristics, diagnosis, and the primary procedure that was performed, there remained 203 pairs of patients in the NS and VS matched group and 203 pairs of patients in the NS and GS matched group. Additional analyses (Table 3) showed no change in length of hospitalization, frequency of complications, discharge with continued care, return to the OR, readmission, or mortality within 30 days between different surgical specialties. These findings, which represent a broad range of demographic features, preoperative variables, baseline comorbidities, and intraoperative factors, suggest no differences in outcomes for patients who undergo carotid endarterectomies performed by neurosurgeons, vascular surgeons, or general surgeons.

\section{Limitations of the Study}

Although the NSQIP collects clinical data prospectively from more than 600 institutions across the US, this is a retrospective analysis. Thus it is not possible to definitively confirm a cause-and-effect relationship between surgical specialty and the outcomes measured. Given the nature of the study, there are potential preoperative variables of interest, including degree of stenosis and symptomatic versus asymptomatic stenosis, that are not available. This is not a randomized study; thus, despite matching on propensity scores, it is not possible to rule out the hypothesis that NS, VS, and GS patients are different from each other with regard to preoperative factors of which we are not aware. We investigated short-term, 30-day postoperative outcomes only; caution should be used to extrapolate beyond this time frame.

Classification of the surgical specialty as NS, VS, or GS was based on the specialty of the attending surgeon. It does not account for fellowship training or experience as a surgeon, which may influence outcomes. ${ }^{3}$ Thus, it was not possible to determine whether outcomes differ between general surgeons, vascular surgeons, fellowship-trained cerebrovascular neurosurgeons, and general neurosurgeons.

CPT codes are an imperfect way to capture the exact details of each surgery-rather, they are useful in quantifying the general nature of the procedures performed. For example, there are no data available on intraoperative use of vascular shunts versus no shunts or use of a vascular patch. However, matching patients on both CPT procedure codes and ICD-9 diagnosis codes to analogous patients by surgeon specialty, we have been able to accurately and reliably identify any adverse outcomes in either group (Table 3 ). The NSQIP database consists of prospectively collected data from both academic and nonacademic hospitals across the country. NSQIP data collection is conducted accurately and precisely, in a standardized fashion, with yearly quality checks that achieve $>95 \%$ reliability for 30-day outcomes in NS, VS, and GS patients after carotid endarterectomy across a wide spectrum of perioperative variables and postoperative outcomes.

\section{Conclusions}

We compared 30-day outcomes in patients undergoing 
carotid endarterectomy between patients operated on by neurosurgeons, vascular surgeons, and general surgeons. In the unmatched cohort, NS patients were more likely than VS and GS patients to undergo endarterectomy for occlusion and stenosis of the carotid artery with cerebral infarct, to have surgery with general anesthesia, to undergo multiple procedures, and to have longer duration of surgery (Table 1). NS patients in the full sample also had slightly increased odds for major complications and transfusions, and nearly 3 times the odds for minor complications, prolonged LOS, and discharge with continued care. However, in the propensity-matched analyses, which controlled carefully for diagnosis and operation as well as baseline and intraoperative patient factors, no differences in outcomes were found. Using a large, prospectively collected, multiinstitutional sample database, our analysis suggests that surgeon specialty is not associated with complications, LOS, discharge with continued care, return to the OR, 30-day readmission, or death.

\section{References}

1. AbuRahma AF, Stone PA, Srivastava M, Hass SM, Mousa AY, Dean LS, et al: The effect of surgeon's specialty and volume on the perioperative outcome of carotid endarterectomy. J Vasc Surg 58:666-672, 2013

2. Ahmed S, Elsheikh M, Stratton IM, Page RC, Adams CB, Wass JA: Outcome of transphenoidal surgery for acromegaly and its relationship to surgical experience. Clin Endocrinol (Oxf) 50:561-567, 1999

3. Allen WC: The relationship between residency programs and fellowships in the educational setting. Clin Orthop Relat Res (257):57-60, 1990

4. American College of Surgeons: Data collection, analysis, and reporting. FACS.org. (https://www.facs.org/qualityprograms/acs-nsqip/program-specifics/data) [Accessed April 6, 2018]

5. Angus DC, van der Poll T: Severe sepsis and septic shock. $\mathbf{N}$ Engl J Med 369:2063, 2013

6. Austin PC: Propensity-score matching in the cardiovascular surgery literature from 2004 to 2006: a systematic review and suggestions for improvement. J Thorac Cardiovasc Surg 134:1128-1135, 2007

7. Austin PC: The use of propensity score methods with survival or time-to-event outcomes: reporting measures of effect similar to those used in randomized experiments. Stat Med 33:1242-1258, 2014

8. Bergstralh EJ, Kosanke JL: Computerized Matching of Cases to Controls. Technical Report Number 56. Rochester, MN: Mayo Clinic, 1995 (http://www.mayo.edu/research/ documents/biostat-56pdf/doc-10026923) [Accessed April 6, 2018]

9. Brott TG, Hobson RW II, Howard G, Roubin GS, Clark WM, Brooks W, et al: Stenting versus endarterectomy for treatment of carotid-artery stenosis. N Engl J Med 363:11-23, 2010

10. Cowan JA Jr, Dimick JB, Thompson BG, Stanley JC, Upchurch GR Jr: Surgeon volume as an indicator of outcomes after carotid endarterectomy: an effect independent of specialty practice and hospital volume. J Am Coll Surg 195:814-821, 2002

11. Diggle P, Liang KY, Zeger SL: Longitudinal Data Analysis. New York: Oxford University Press, 1994

12. Enomoto LM, Hill DC, Dillon PW, Han DC, Hollenbeak CS: Surgical specialty and outcomes for carotid endarterectomy: evidence from the National Surgical Quality Improvement Program. J Surg Res 188:339-348, 2014

13. Feasby TE, Kennedy J, Quan H, Ghali WA: Outcomes in carotid endarterectomy performed by vascular surgeons or neurosurgeons. Stroke 33:1458, 2002

14. Ferguson GG, Eliasziw M, Barr HW, Clagett GP, Barnes RW, Wallace MC, et al: The North American Symptomatic Carotid Endarterectomy Trial: surgical results in 1415 patients. Stroke 30:1751-1758, 1999

15. French B, Farjah F, Flum DR, Heagerty PJ: A general framework for estimating volume-outcome associations from longitudinal data. Stat Med 31:366-382, 2012

16. Halm EA, Hannan EL, Rojas M, Tuhrim S, Riles TS, Rockman CB, et al: Clinical and operative predictors of outcomes of carotid endarterectomy. J Vasc Surg 42:420-428, 2005

17. Hannan EL, Popp AJ, Feustel P, Halm E, Bernardini G, Waldman J, et al: Association of surgical specialty and processes of care with patient outcomes for carotid endarterectomy. Stroke 32:2890-2897, 2001

18. Hollenbeak CS, Bowman AR, Harbaugh RE, Casale PN, Han D: The impact of surgical specialty on outcomes for carotid endarterectomy. J Surg Res 159:595-602, 2010

19. Hussain MA, Mamdani M, Tu JV, Saposnik G, Salata K, Bhatt DL, et al: Association between operator specialty and outcomes after carotid artery revascularization. J Vasc Surg 67:478-489, 489.e1-489.e6, 2018

20. Kempczinski RF, Brott TG, Labutta RJ: The influence of surgical specialty and caseload on the results of carotid endarterectomy. J Vasc Surg 3:911-916, 1986

21. Khuri SF, Henderson WG, Daley J, Jonasson O, Jones RS, Campbell DA Jr, et al: The patient safety in surgery study: background, study design, and patient populations. J Am Coll Surg 204:1089-1102, 2007

22. Lieber BA, Henry JK, Agarwal N, Day JD, Morris TW III, Stephens ML, et al: Impact of surgical specialty on outcomes following carotid endarterectomy. Neurosurgery 80:217225, 2017

23. Nicoll D, McPhee S, Pignone M, Mark L: Pocket Guide to Diagnostic Tests, ed 6. New York: McGraw-Hill, 2010

24. O'Neill L, Lanska DJ, Hartz A: Surgeon characteristics associated with mortality and morbidity following carotid endarterectomy. Neurology 55:773-781, 2000

25. Ringleb PA, Allenberg J, Brückmann H, Eckstein HH, Fraedrich G, Hartmann M, et al: 30 day results from the SPACE trial of stent-protected angioplasty versus carotid endarterectomy in symptomatic patients: a randomised non-inferiority trial. Lancet 368:1239-1247, 2006

26. Rosenbaum PR: Coherence in observational studies. Biometrics 50:368-374, 1994

27. Rosenbaum PR, Rubin DB: The central role of the propensity score in observational studies for causal effects. Biometrika 70:41-55, 1983

28. Roubin GS, New G, Iyer SS, Vitek JJ, Al-Mubarak N, Liu MW, et al: Immediate and late clinical outcomes of carotid artery stenting in patients with symptomatic and asymptomatic carotid artery stenosis: a 5-year prospective analysis. Circulation 103:532-537, 2001

29. Ruby ST, Robinson D, Lynch JT, Mark H: Outcome analysis of carotid endarterectomy in Connecticut: the impact of volume and specialty. Ann Vasc Surg 10:22-26, 1996

30. Schmidt CM, Turrini O, Parikh P, House MG, Zyromski NJ, Nakeeb A, et al: Effect of hospital volume, surgeon experience, and surgeon volume on patient outcomes after pancreaticoduodenectomy: a single-institution experience. Arch Surg 145:634-640, 2010

31. Seicean A, Alan N, Seicean S, Neuhauser D, Benzel EC, Weil RJ: Surgeon specialty and outcomes after elective spine surgery. Spine (Phila Pa 1976) 39:1605-1613, 2014

32. Shah DM, Darling RC, Chang BB, Paty PS, Kreienberg PB, Roddy SP, et al: Analysis of factors contributing to improved outcome for carotid endarterectomy. Semin Vasc Surg 17:257-259, 2004 
33. Shiloach M, Frencher SK Jr, Steeger JE, Rowell KS, Bartzokis $\mathrm{K}$, Tomeh MG, et al: Toward robust information: data quality and inter-rater reliability in the American College of Surgeons National Surgical Quality Improvement Program. J Am Coll Surg 210:6-16, 2010

34. Witt PD, Wahlen JC, Marsh JL, Grames LM, Pilgram TK:

The effect of surgeon experience on velopharyngeal functional outcome following palatoplasty: is there a learning curve? Plast Reconstr Surg 102:1375-1384, 1998

\section{Disclosures}

The authors report no conflict of interest concerning the materials or methods used in this study or the findings specified in this paper.

\section{Author Contributions}

Conception and design: A Seicean, Weil. Acquisition of data: A Seicean, Kumar. Analysis and interpretation of data: all authors. Drafting the article: A Seicean, Kumar. Critically revising the article: all authors. Reviewed submitted version of manuscript: all authors. Approved the final version of the manuscript on behalf of all authors: A Seicean. Statistical analysis: A Seicean, Kumar.

\section{Supplemental Information}

Online-Only Content

Supplemental material is available with the online version of the article.

Supplemental Tables 1 and 2. https://thejns.org/doi/ suppl/10.3171/2018.2.JNS173014.

\section{Previous Presentations}

Results of this work were presented, in part, as an oral presentation at the 2014 annual conference of the American Association of Neurological Surgeons in San Francisco, CA.

\section{Correspondence}

Andreea Seicean: University of Illinois at Chicago, IL. andreea. seicean@gmail.com. 